\title{
Eigensolutions of the Schrödinger equation with a class of Yukawa potentials via supersymmetric approach
}

\author{
C. A. Onate ${ }^{1} \cdot$ J. O. Ojonubah ${ }^{2}$
}

Received: 15 August 2015/ Accepted: 12 October 2015/Published online: 20 November 2015

(C) The Author(s) 2015. This article is published with open access at Springerlink.com

\begin{abstract}
Using the basic concept of the supersymmetric shape invariance approach and formalism, we obtained an approximate solution of the Schrödinger equation with an interaction of inversely quadratic Yukawa potential, Yukawa potential and Coulomb potential which we considered as a class of Yukawa potentials. By varying the potential strengths, we obtained a solution for Hellmann potential, Yukawa potential, Coulomb potential and inversely quadratic Yukawa potential. The numerical results we obtained show that the interaction of these potentials is equivalent to each of the potential.
\end{abstract}

Keywords Schrödinger equation · Eigensolutions · Class of Yukawa potentials

\section{Introduction}

In the recent time, physicists have developed much interest in searching for the exponential-type potentials. The reason is that most of the exponential-type potentials play an important role in physics, e.g. Yukawa potential is used in plasma, solid-state and atomic physics [1]. As a result, many authors have solved both relativistic and non-relativistic wave equations with these potentials. For instance, Zhang et al. [2] obtained approximate solutions of the Schrödinger equation with the Generalized Morse potential

C. A. Onate

oaclems14@physicist.net; onateca12@gm.us

1 Theoretical Physics Section, Department of Physics, University of Benin, Benin, Nigeria

2 Mathematics Department, Federal College of Education Okene, Okene, Nigeria model including a centrifugal term. Jia et al. [3] solved sixparameter exponential-type potentials. Onate [4] obtained relativistic and non-relativistic solutions of the inversely quadratic Yukawa potential. Falaye et al. [5] obtained bound state solutions of the Schrödinger equation with Manning-Rosen potential. Hassanabadi et al. [6, 7] obtained Actual and general Manning-Rosen potential under spin and pseudospin symmetries of the Dirac equation; approximate solutions of the Schrödinger equation under Manning-Rosen potential in arbitrary dimensions via SUSY QM. Hamzavi et al. [8] obtained approximate spin and pseudospin solutions of the Dirac equation for inversely quadratic Yukawa potential and tensor interaction. Maghsoodi et al. [1] solved Dirac particles in the presence of Yukawa potential plus a tensor interaction in SUSY QM frame work. Ikhdair [9] obtained on the bound state solutions of the Manning-Rosen potential including an improved approximation to the orbital centrifugal term.

The solutions of the wave equations (either Schrödinger, Klein-Gordon, Dirac or D.K.P.) with any of these exponential-type potentials are obtained using different methods which include: asymptotic iteration method (AIM) [1016], Nikiforov-Uvarov (N.U) method [17-20], exact/ proper quantization rule [21], supersymmetric method [2227], $1 / N$ shifted expansion method [28], etc.

Motivated by the success in the exponential-type potentials, we attempt to investigate the solutions of the radial Schrödinger equation with a class of Yukawa potentials given as

$V(r)=\frac{-b r+r c e^{-\delta r}-a e^{-2 \delta r}}{r^{2}}$,

where $a, b$ and $c$ are potential strength and $\delta$ is the screening parameter. The potential is obtained by the addition of Hellmann potential and inversely quadratic 
Yukawa potential which is equivalent to the interaction of inversely quadratic Yukawa potential, Coulomb potential and Yukawa potential. This potential has its application where its components are useful. It is noted that the exact solution of the radial Schrödinger equation with potential (1) is not possible due to the presence of the inverse square term. Therefore, to obtain an approximate solutions, we employ a suitable approximation scheme. It is found that such approximation proposed by Greene and Aldrich [29]

$\frac{1}{r^{2}}=\frac{\delta^{2}}{\left(1-e^{-\delta r}\right)^{2}}$.

is a good approximation to the centrifugal/inverse square term which is valid for $\delta \ll 1$ for a short potential range.

Our work is arranged as follows: in the following section, we obtain the bound state solutions. We present discussion and concluding remarks at the end of the article.

\section{Bound state solutions}

To study any quantum physical system, we solve the original Schrödinger equation given as [9, 30, 31]

$$
\left(\frac{P^{2}}{2 \mu}+V(r)\right) \psi_{n, \ell, m}(r)=E_{n, \ell} \psi_{n, \ell, m}(r)
$$

where $E_{n, \ell}$ is the energy, $\mu$ is the particle mass and $V(r)$ is the potential. Setting the wave functions $\psi_{n, \ell, m}(r)=\frac{R_{n, \ell}(r) Y_{\ell, m}(\theta, \varphi)}{r}$, we obtained the following radial Schrödinger equation

$\left[\frac{\mathrm{d}^{2}}{\mathrm{~d} r^{2}}+\frac{2 \mu}{\hbar^{2}}\left(E_{n, \ell}-V(r)\right)-\frac{\ell(\ell+1)}{r^{2}}\right] R_{n, \ell}(r)=0$.

With Eqs. (1) and (2), (4) becomes

$\frac{\mathrm{d}^{2} R_{n, \ell}(r)}{\mathrm{d} r^{2}}=\left[V_{\mathrm{eff}}-E_{\mathrm{eff}}\right] R_{n, \ell}(r)$,

where $\quad V_{\mathrm{eff}}=\left[\frac{2 \mu \delta}{\hbar^{2}}(a \delta-b+c)+\ell(\ell+1) \delta^{2}\right] \frac{e^{-\delta r}}{1-e^{-\delta r}}-$ $\left[\frac{2 \mu a \delta^{2}}{\hbar^{2}}-\ell(\ell+1) \delta^{2}\right] \frac{e^{-\delta r}}{\left(1-e^{-\delta r}\right)^{2}}$ and $-E_{\text {eff }}=-\frac{2 \mu \delta}{\hbar^{2}}\left(E_{n, \ell}+b\right)$ $+\ell(\ell+1) \delta^{2}$. Since we are dealing with a Schrödinger-like equation that we solved by means of SUSY QM [6, 32-35], the first step in the SUSY approach is finding the solution of the Riccati equation [6]. Using the shape invariance formalism, it can be easily seen that

$W^{2}(r)-\frac{\mathrm{d} W(r)}{\mathrm{d} r}=V_{\mathrm{eff}}-E_{\text {eff }}$,

whose solution is given as

$W(r)=A-\frac{B e^{-\delta r}}{1-e^{-\delta r}}$,
The ground state wave function $R_{0, \ell}(r)$ as

$R_{0, \ell}(r)=\exp \left(-\int W(r) \mathrm{d} r\right)$

where $W(r)$ is called the superpotential function in supersymmetric quantum mechanics [36-38]. Substitute Eq. (7) into Eq. (6), we deduce the following relations

$A^{2}=-E_{\mathrm{eff}}$

$B=\frac{2 \mu a \delta}{\hbar^{2}}-(\ell+1) \delta$,

$A=\frac{2 \mu \delta(b-c-a \delta)-\ell(\ell+1) \delta^{2}-B^{2}}{2 B}$.

With Eq. (7), we can construct the two partner potentials as follows:

$$
\begin{aligned}
U_{+} & =W^{2}(r)+\frac{\mathrm{d} W(r)}{\mathrm{d} r} \\
& =A^{2}-\frac{B(B+2 A) e^{-\delta r}}{1-e^{-\delta r}}+\frac{B(B+\delta) e^{-\delta r}}{\left(1-e^{-\delta r}\right)^{2}} \\
U_{+} & =W^{2}(r)+\frac{\mathrm{d} W(r)}{\mathrm{d} r} \\
& =A^{2}-\frac{B(B+2 A) e^{-\delta r}}{1-e^{-\delta r}}+\frac{B(B-\delta) e^{-\delta r}}{\left(1-e^{-\delta r}\right)^{2}} .
\end{aligned}
$$

Using the shape invariance technique [6, 39-42], it can readily be shown that the two partner potentials are shape invariant. Therefore, their relationship is written as

$U_{+}\left(r, a_{0}\right)=U_{-}\left(r, a_{1}\right)+T\left(a_{1}\right)$,

where $a_{0}$ is an old set of parameters in which the new set of parameters $a_{1}$ is obtained from and $T\left(a_{1}\right)$ is a remainder that is independent of the variable $r$. Here, $B=a_{0}$ via mapping of the form $a_{1} \rightarrow a_{0}-\delta, \quad a_{2} \rightarrow a_{0}-2 \delta, \quad a_{3} \rightarrow a_{0}-3 \delta$. Thus, a generalization is drawn as $a_{n} \rightarrow a_{0}-n \delta$. In terms of the parameters of the problem, we obtain the following relations:

$$
\begin{aligned}
T\left(a_{1}\right)= & {\left[\frac{2 \mu \delta(b-c-a \delta)-\ell(\ell+1) \delta^{2}-a_{0}^{2}}{2 a_{0}}\right] } \\
& -\left[\frac{2 \mu \delta(b-c-a \delta)-\ell(\ell+1) \delta^{2}-a_{1}^{2}}{2 a_{1}}\right], \\
T\left(a_{2}\right)= & {\left[\frac{2 \mu \delta(b-c-a \delta)-\ell(\ell+1) \delta^{2}-a_{1}^{2}}{2 a_{1}}\right] } \\
& -\left[\frac{2 \mu \delta(b-c-a \delta)-\ell(\ell+1) \delta^{2}-a_{2}^{2}}{2 a_{2}}\right], \\
T\left(a_{3}\right)= & {\left[\frac{2 \mu \delta(b-c-a \delta)-\ell(\ell+1) \delta^{2}-a_{2}^{2}}{2 a_{2}}\right] } \\
& -\left[\frac{2 \mu \delta(b-c-a \delta)-\ell(\ell+1) \delta^{2}-a_{3}^{2}}{2 a_{3}}\right],
\end{aligned}
$$




$$
\begin{aligned}
T\left(a_{n}\right)= & {\left[\frac{2 \mu \delta(b-c-a \delta)-\ell(\ell+1) \delta^{2}-a_{n-1}^{2}}{2 a_{n-1}}\right] } \\
& -\left[\frac{2 \mu \delta(b-c-a \delta)-\ell(\ell+1) \delta^{2}-a_{n}^{2}}{2 a_{n}}\right],
\end{aligned}
$$

The energy spectral can then be determine as follows: $E_{0, \ell}^{-}=0$,

$$
\begin{aligned}
E_{n, \ell}^{-} & =E_{\mathrm{eff}}+E_{0, \ell}^{-}=\sum_{k=1}^{n} T\left(a_{k}\right) \\
& =\left[\frac{2 \mu \delta(b-c-a \delta)-\ell(\ell+1) \delta^{2}-a_{n}^{2}}{2 a_{n}}\right] .
\end{aligned}
$$

This gives energy equation as

$$
\begin{aligned}
E_{n, \ell}= & \delta\left(\frac{\delta \hbar^{2} \ell(\ell+1)}{2 \mu}-b\right) \\
& -\frac{\delta^{2} \hbar^{2}}{2 \mu}\left[\frac{\frac{2 \mu}{\delta \hbar^{2}}(b-c-a \delta)-(n+\ell+1)^{2}-\ell(\ell+1)}{2(\ell+n+1)}\right]^{2} .
\end{aligned}
$$

\section{Wave function}

To obtain the un-normalized wave function, we define a variable of the form $y=e^{-\delta r}$ and substitute it into Eq. (5) to have

$\frac{\mathrm{d}^{2} R_{n, \ell}(r)}{\mathrm{d} y^{2}}+\frac{1}{y} \frac{\mathrm{d} R_{n, \ell}(r)}{\mathrm{d} y}+\frac{N y^{2} Q y+P}{(y(1-y))^{2}} R_{n, \ell}(r)=0$,

where

$$
\begin{aligned}
& P=\frac{2 \mu\left(b \delta+E_{n, \ell}\right)}{\delta^{2} \hbar^{2}}-\ell(\ell+1), \\
& Q=-\frac{2 \mu\left(b \delta+c \delta+E_{n, \ell}\right)}{\delta^{2} \hbar^{2}}, \\
& N=\frac{2 \mu\left(a \delta+c \delta^{2}+2 E_{n, \ell}\right)}{\delta^{2} \hbar^{2}}
\end{aligned}
$$

Analyzing the asymptotic behavior of Eq. (22) at origin and at infinity, it can be tested that when $r \rightarrow 0(y \rightarrow 1)$ and when $r \rightarrow \infty(y \rightarrow 0)$ Eq. (22) has a solution

$$
R_{n, \ell}(y)=y^{\eta}(1-y)^{\varepsilon} \text {, }
$$

where

$$
\begin{aligned}
& \eta=\sqrt{-\frac{2 \mu\left(b \delta+E_{n, \ell}\right)}{\delta^{2} \hbar^{2}}+\ell(\ell+1)}, \\
& \varepsilon=\frac{1}{2}+\frac{1}{2} \sqrt{1-\frac{2 \mu a}{\delta \hbar^{2}}+\ell(\ell+1)} .
\end{aligned}
$$

Now, taking a trial wave function of the form $R_{n, \ell}(y)=$ $y^{\eta}(1-y)^{\varepsilon} f(y)$ and substitute it into Eq. (22), we have

$$
\begin{aligned}
& f^{\prime \prime}(y)+f^{\prime}(y) \frac{2 \eta+1-y(2 \eta+2 \varepsilon+1)}{y(1-y)}+f(y) \frac{(\eta+\varepsilon)^{2}+N}{y(1-y)} \\
& \quad=0 .
\end{aligned}
$$

Equation (27) is a differential equation satisfied by the hypergeometric function. Thus, its solution is obtained as:

$f(y)={ }_{2} F_{1}(-n, n+2(\eta+\varepsilon) ; 2 \eta+1, y)$.

Replacing the function $f(y)$ with the hypergeometric function and write a complete radial wave function as:

$R_{n, \ell}(y)=y^{\eta}(1-y)^{\varepsilon}{ }_{2} F_{1}(-n, n+2(\eta+\varepsilon) ; 2 \eta+1, y)$.

\section{Results and discussion}

Some special cases of interest are studied here, when $a=0$, our potential (1) reduces to Hellmann potential

$V(r)=\frac{-b+c e^{-\delta r}}{r}$,

which reduces the energy Eq. (21) to

$$
\begin{aligned}
E_{n, \ell}= & \delta\left(\frac{\delta \hbar^{2} \ell(\ell+1)}{2 \mu}-b\right) \\
& -\frac{\delta^{2} \hbar^{2}}{2 \mu}\left[\frac{\frac{2 \mu}{\delta \hbar^{2}}(b-c)-(n+\ell+1)^{2}-\ell(\ell+1)}{2(\ell+n+1)}\right]^{2} .
\end{aligned}
$$

When $a=c=0$, the potential (1) reduces to Coulomb potential

$V(r)=\frac{-b}{r}$,

and the energy Eq. (21) turns to

$$
\begin{aligned}
E_{n, \ell}= & \delta\left(\frac{\delta \hbar^{2} \ell(\ell+1)}{2 \mu}-b\right) \\
& -\frac{\delta^{2} \hbar^{2}}{2 \mu}\left[\frac{\frac{2 \mu b}{\delta \hbar^{2}}-(n+\ell+1)^{2}-\ell(\ell+1)}{2(\ell+n+1)}\right]^{2} .
\end{aligned}
$$

When $a=b=0$, the potential (1) reduces to Yukawa potential

$V(r)=\frac{-c e^{-\delta r}}{r}$,

and the energy Eq. (21) turns to 
Table 1 Bound states energy for the class of Yukawa potential $E_{n, \ell}^{\mathrm{CYP}}$, Hellmann potential $E_{n, \ell}^{\mathrm{HP}}$, Coulomb potential $E_{n, \ell}^{\mathrm{CP}}$ and Yukawa potential $E_{n, \ell}^{\mathrm{YP}}$

\begin{tabular}{llllll}
\hline State & $\delta$ & $E_{n, \ell}^{\mathrm{CYP}}$ & $E_{n, \ell}^{\mathrm{HP}}$ & $E_{n, \ell}^{\mathrm{CP}}$ & $E_{n, \ell}^{\mathrm{YP}}$ \\
\hline $2 \mathrm{p}$ & 0.001 & -2.2474553 & -2.2475002 & -2.2485002 & -2.2455002 \\
& 0.005 & -2.2374615 & -2.2375062 & -2.2425062 & -2.2275062 \\
& 0.010 & -2.2249805 & -2.2250250 & -2.2350250 & -2.2050250 \\
$3 \mathrm{p}$ & 0.001 & -0.9983214 & -0.9983347 & -0.9993347 & -0.9963347 \\
& 0.005 & -0.9916874 & -0.9917007 & -0.9967007 & -0.9817007 \\
& 0.010 & -0.9834563 & -0.9834694 & -0.9934694 & -0.9634694 \\
$3 \mathrm{~d}$ & 0.001 & -0.9969870 & -0.9970003 & -0.9980003 & -0.9950003 \\
& 0.005 & -0.9849930 & -0.9850063 & -0.9900063 & -0.9750063 \\
& 0.010 & -0.9700120 & -0.9700250 & -0.9800250 & -0.9500250 \\
$4 \mathrm{p}$ & 0.001 & -0.5611224 & -0.5611281 & -0.5621281 & -0.5591281 \\
& 0.005 & -0.5556960 & -0.5557016 & -0.5607016 & -0.5457016 \\
& 0.010 & -0.5490507 & -0.5490563 & -0.5590563 & -0.5290563 \\
$4 \mathrm{~d}$ & 0.001 & -0.5603710 & -0.5603766 & -0.5613766 & -0.5583766 \\
& 0.005 & -0.5519085 & -0.5519141 & -0.5569141 & -0.5419141 \\
& 0.010 & -0.5414008 & -0.5414063 & -0.5514063 & -0.5214063 \\
$4 \mathrm{f}$ & 0.001 & -0.5592447 & -0.5592503 & -0.5602503 & -0.5572503 \\
& 0.005 & -0.5462508 & -0.5462563 & -0.5572563 & -0.5362563 \\
& 0.010 & -0.5300196 & -0.5300250 & -0.5400250 & -0.5100250 \\
\hline \multirow{3}{*}{} & & & & &
\end{tabular}

$$
\begin{aligned}
E_{n, \ell}= & \frac{\delta^{2} \hbar^{2} \ell(\ell+1)}{2 \mu} \\
& -\frac{\delta^{2} \hbar^{2}}{2 \mu}\left[\frac{\frac{-2 \mu c}{\delta \hbar^{2}}-(n+\ell+1)^{2}-\ell(\ell+1)}{2(\ell+n+1)}\right]^{2} .
\end{aligned}
$$

When $b=c=0$, potential (1) reduces to inversely quadratic Yukawa potential

$V(r)=\frac{-a e^{-2 \delta r}}{r^{2}}$

and the energy Eq. (21) turns to

$$
\begin{aligned}
E_{n, \ell}= & \frac{\delta^{2} \hbar^{2} \ell(\ell+1)}{2 \mu} \\
& -\frac{\delta^{2} \hbar^{2}}{2 \mu}\left[\frac{\frac{-2 \mu a}{\hbar^{2}}-(n+\ell+1)^{2}-\ell(\ell+1)}{2(\ell+n+1)}\right]^{2} .
\end{aligned}
$$

In Table 1, we numerically reported the energy eigenvalues for a class of Yukawa potential $\left(a=1 \times 10^{-5}, b=2 \times 10^{0}, \quad c=-1 \times 10^{0}\right)$, Hellmann potential $\left(a=0, b=2 \times 10^{0}, c=-1 \times 10^{0}\right)$, Coulomb potential $(a=0=c=0, \quad b=3)$ and Yukawa potential $\left(a=b=0, c=-3 \times 10^{0}\right.$ ) for $2 \mathrm{p}, 3 \mathrm{p}, 3 \mathrm{~d}, 4 \mathrm{p}, 4 \mathrm{~d}$ and $4 \mathrm{f}$. In Table 2, we have reported the energy eigenvalues of these potentials for $n=0, \quad \in \ell=0 ; n=1, \quad \ell=0,1$; $n=2, \ell=0,1,2 ; n=3, \in \ell=0,1,2,3$ and $n=4, \ell=$ $0,1,2,3,4$. In Table 1 , energy increases as the screening
Table 2 Bound states energy for the class of Yukawa potential $E_{n, \ell}^{\mathrm{CYP}}$, Hellmann potential $E_{n, \ell}^{\mathrm{HP}}$, Coulomb potential $E_{n, \ell}^{\mathrm{CP}}$ and Yukawa potential $E_{n, \ell}^{\mathrm{YP}}$

\begin{tabular}{llllll}
\hline$n$ & $\ell$ & $E_{n, \ell}^{\mathrm{CYP}}$ & $E_{n, \ell}^{\mathrm{HP}}$ & $E_{n, \ell}^{\mathrm{CP}}$ & $E_{n, \ell}^{\mathrm{YP}}$ \\
\hline 0 & 0 & -8.9896656 & -8.9900250 & -9.0000250 & -8.9700250 \\
1 & 0 & -2.2400552 & -2.2401000 & -2.2501000 & -2.2201000 \\
& 1 & -0.9834563 & -0.9834694 & -0.9934694 & -0.9634694 \\
2 & 0 & -0.9902117 & -0.9902250 & -1.0002250 & -0.9702250 \\
& 1 & -0.5490507 & -0.5490563 & -0.5590563 & -0.5290563 \\
& 2 & -0.3431582 & -0.3431610 & -0.3531610 & -0.3231610 \\
3 & 0 & -0.5528944 & -0.5529000 & -0.5629000 & -0.5329000 \\
& 1 & -0.3481262 & -0.3481290 & -0.3581290 & -0.3281290 \\
& 2 & -0.2356234 & -0.2356250 & -0.2456250 & -0.2156250 \\
& 3 & -0.1670240 & -0.1670250 & -0.1770250 & -0.1470250 \\
4 & 0 & -0.3506222 & -0.3506250 & -0.3606250 & -0.3306250 \\
& 1 & -0.2391345 & -0.2391361 & -0.2491361 & -0.2191361 \\
& 2 & -0.1709424 & -0.1709434 & -0.1809434 & -0.1509434 \\
& 3 & -0.1260556 & -0.1260563 & -0.1360563 & -0.1060563 \\
& 4 & -0.0948517 & -0.0948522 & -0.1048522 & -0.0748522 \\
\hline
\end{tabular}

parameter increases. In Table 2, energy increases as $n$ increases. In Table 3 , we compared our result for the Hellmann potential with the result from two other methods. 
Table 3 Ro-vibrational energy spectrum $\left(-E_{n, \ell}\right)$ for the Hellmann potential with $2 m=\hbar=1, b=2$ and $c=1$

\begin{tabular}{lllll}
\hline State & $\delta$ & SUSY & NU [43] & AP [43] \\
\hline $2 \mathrm{p}$ & 0.001 & 0.063999 & 0.064000 & 0.063495 \\
& 0.005 & 0.069975 & 0.070000 & 0.067377 \\
& 0.010 & 0.077400 & 0.077500 & 0.072020 \\
$3 \mathrm{p}$ & 0.001 & 0.029499 & 0.029279 & 0.028765 \\
& 0.005 & 0.036356 & 0.035309 & 0.032480 \\
& 0.010 & 0.044869 & 0.042903 & 0.036645 \\
$3 \mathrm{~d}$ & 0.001 & 0.029274 & 0.029388 & 0.028767 \\
& 0.005 & 0.035184 & 0.035817 & 0.032526 \\
& 0.010 & 0.042403 & 0.043825 & 0.036814 \\
$4 \mathrm{p}$ & 0.001 & 0.017436 & 0.017128 & 0.016602 \\
& 0.005 & 0.024652 & 0.023200 & 0.020100 \\
& 0.010 & 0.033606 & 0.030925 & 0.023711 \\
$4 \mathrm{~d}$ & 0.001 & 0.017308 & 0.017180 & 0.016604 \\
& 0.005 & 0.023952 & 0.023464 & 0.020142 \\
& 0.010 & 0.032056 & 0.031256 & 0.023857 \\
& 0.001 & 0.017117 & 0.017311 & 0.016607 \\
$4 \mathrm{f}$ & 0.005 & 0.022925 & 0.024027 & 0.020206 \\
& 0.010 & 0.029825 & 0.032356 & 0.024072 \\
\hline & & & & \\
& & & &
\end{tabular}

\section{Conclusions}

We have obtained approximate solutions of the Schrödinger equation by combining inversely quadratic Yukawa potential, Yukawa potential and Coulomb potential. We deduced that from the energy equation of these combined potentials, the energy equation of Hellmann potential as well as these individual potential can be obtained. In Tables 1 and 2, we have numerically reported the equivalence of the energy of these potentials.

Open Access This article is distributed under the terms of the Creative Commons Attribution 4.0 International License (http://creative commons.org/licenses/by/4.0/), which permits unrestricted use, distribution, and reproduction in any medium, provided you give appropriate credit to the original author(s) and the source, provide a link to the Creative Commons license, and indicate if changes were made.

\section{References}

1. Maghsoodi, E., Hassanabadi, E., Aydoğdu, O.: Dirac particles in the presence of the Yukawa potential plus a tensor interaction in SUSYQM framework. Phys. Scr. 86, 015005 (2012)

2. Zhang, L.H., Li, X.P., Jia, C.S.: Approximate solutions of the Schrödinger equation with the generalized Morse potential model including the centrifugal term. Int. J. Quantum Chem. 111, 1870 (2011)

3. Jia, C.S., Zeng, X.L., Li, S.C., Sun, L.T., Yang, Q.B.: Six parameter exponential-type potential and the identity for the exponential-type potentials. Commun. Theor. Phys. 37, 523 (2002)
4. Onate, C.A.: Relativistic and Non-relativistic Solutions of the Inversely Quadratic Yukawa potential. Afr. Rev. Phys. 8, 0046 (2013)

5. Falaye, B.J., Oyewumi, K.J., Ibrahim, T.T., Punyasena, M.A., Onate, C.A.: Bound state solutions of the Manning-Rosen potential. Can. J. Phys. 91, 98 (2013)

6. Hassanabadi, H., Maghsoodi, E., Zarrinkamar, S., Rahimov, H.: Actual and general Manning-Rosen potentials under spin and speudospin symmetries of the Dirac equation. Can. J. Phys. 90, 633 (2012)

7. Hassanabadi, H., Lu, L.L., Zarrinkamar, S., Liu, G., Rahimov, H.: Appropriate solutions of Schrodinger equation under ManningRosen potential in arbitrary dimensions via SUSYQM. Acta Phys. Pol. A 122, 650 (2012)

8. Hamzavi, M., Ikhdair, S.M., Ita, B.I.: Approximate spin and pseudospin solutions to the Dirac equation for the inversely quadratic Yukawa potential and tensor interaction. Phys. Scr. 85, 045009 (2012)

9. Ikhdair, S.M.: On the bound state solutions of the Manning-Rosen potential including an improved approximation to the orbital centrifugal term. Phys. Scr. 83, 015010 (2011)

10. Bayrak, O., Boztosun, I.: Bound state solutions of the Hulthen potential by using the asymptotic iteration method. Phys. Scr. 76, $92(2007)$

11. Soylu, A., Bayrak, O., Boztosun, I.: $\kappa$ state solutions of the Dirac equation for the Eckart potential with pseudospin and spin symmetry. J. Phys. A: Math. Theor. 41, 065308 (2008)

12. Bayrak, O., Boztosun, I.: The pseudospin symmetric solution of the Morse potential for any $\kappa$ state. J. Phys. A: Math. Theor. 40, 11119 (2007)

13. Soylu, A., Bayrak, O., Boztosun, I.: An approximate solution of Dirac-Hulthen problem with pseudospin and spin symmetry for any $\kappa$ state. J. Math. Phys. 48, 082302 (2007)

14. Oyewumi, K.J., Falaye, B.J., Onate, C.A., Oluwadare, O.J., Yahya, W.A.: Thermodynamic properties and approximate solutions of the Schrodinger equation with the shifted Deng-Fan potential model. Mol. Phys. doi:10.1080/00268976

15. Oyewumi, K.J., Falaye, B.J., Onate, C.A., Oluwadare, O.J., Yahya, W.A.: $\mathrm{k}$ state solutions for the fermionic massive spin-1/2 particles interacting with double ring-shaped Kratzer and Oscillator potentials. Int. J. Mod. Phys. E 23, 1450005 (2014)

16. Behere, S.H.: Anharmonicity constants of diatomic molecules for external Rydberg and Dimitreva-Zenevich potential functions. Indian J. Phys. 55B, 272 (1982)

17. Zarrinkamar, S., Rajabi, A.A., Hassanabadi, H., Rahimov, H.: Analytical treatment of the two-body spinless Salpeter equation with the Hulthen potential. Phys. Scr. 84, 065008 (2011)

18. Satare, M.R., Haidari, S.: Spin symmetry of the Dirac equation with the Yukawa potential. Phys. Scr. 81, 065201 (2010)

19. Ikhdair, S.M., Sever, R.: Two approximation schemes to the bound states of the Dirac-Hulthen problem. J. Phys. A Math. Theor. 44, 355301 (2011)

20. Onate, C.A.: Approximate Solutions of the Non-Relativistic Schrodinger Equation with an Interaction of Coulomb and Hulthen potentials. SOP Trans. Theor. Phys. 1, 118 (2014)

21. Ikhdair, S.M., Hasna, J.A.: Quantization rule solutions to the Hulthen potential in arbitrary dimension with a new approximation scheme for the centrifugal term. Phys. Scr. 83, 025002 (2011)

22. Wei, G.F., Dong, S.H.: Pseudospin symmetry in the relativistic Manning-Rosen potential including a Pekeris-type approximation to the pseudo-centrifugal term. Phys. Lett. B 686, 288 (2010)

23. Hassanabadi, H., Zarrinkamar, S., Rahimov, H.: Approximate solution of D-dimensional Klein-Gordon equation with Hulthentype potential via SUSYQM. Commun. Theor. Phys. 56, 423 (2011) 
24. Jia, C.S., Chen, T., He, S.: Bound state solutions of the KleinGordon equation with the improved expression of the ManningRosen potential energy model. doi:10.1016/physleta.2013.01.016

25. Hassanabadi, H., Maghsoodi, E., Zarrinkamar, S., Rahimov, H.: Actual and general Manning-Rosen potentials under spin and pseudospin symmetries of the Dirac equation. Can. J. Phys. 90, 633 (2012)

26. Hassanabadi, H., Lu, L.L., Zarrinkamar, S., Liu, G., Rahimov, H.: Approximate Solutions of Schrodinger Equation under ManningRosen Potential in Arbitrary Dimension via SUSY MQ. Acta Phys. Pol., A 122, 1111 (2012)

27. Onate, C.A.: Bound state solutions of Duffin-Kemmer-Petaiu equation with Yukawa potential. Afr. Rev. Phys. 9, 0033 (2015)

28. Hammed, R.H.: Approximate Solution of the Schrodinger equation with Manning-Rosen potential in Two Dimensions by using the shifted 1/N expansion method. J. Basrah Res. 38, 51 (2012)

29. Greene, R.L., Aldrich, C.: Variational wave functions for a screened Coulomb potential. Phys. Rev. A 14, 2363 (1976)

30. Schiff, L.I.: Quantum Mechanics, 3rd edn. Mc Graw-Hill, New York (1968)

31. Landau, L.D., Lifshitz, E.M.: Quantum Mechanics: Non-Relativistic Theory, 3rd edn. Pergamon, New York (1977)

32. Zhang, L.H., Li, X.P., Jia, C.S.: Few-Body Syst. 52, 11 (2011)

33. Gendenstein, L.E.: Derivation of Exact Spectra of the Schrodinger Equation by means of supersymmetry. J. Exp. Theor. Phys. Lett. 38, 356 (1983)

34. Hassanabadi, H., Maghsoodi, E., Zarrinkamar, S., Rahimov, H.: An Approximate solution of the Dirac equation for Hyperbolic scalar and vector potentials and a Coulomb tensor interaction by SUSY QM. Mod. Phys. Lett. A 26, 2703 (2011)
35. Zarrinkamar, S., Rajabi, A.A., Hassanabadi, H.: Dirac equation for the harmonic scalar and vector potentials and linear plus coulomb like tensor potential : the SUSY approach. Ann. Phys. 325, 2522 (2010)

36. Cooper, F., Freedman, B.: Aspects of supersymmetric quantum mechanics. Ann. Phys. 146, 262 (1983)

37. Onate, C.A.: An approximate solution of dirac equation for second poschl-teller like scalar and vector potentials with a coulomb tensor interaction. Afr. Rev. Phys. 8, 0020 (2013)

38. Ikot, A.N., Maghsoodi, E., Ibanga, E.J., Zarrinkamar, S., Hassanabadi, H.: Spin and pseudospin symmetries of the Dirac equation with shifted Hulthen potential using supersymmetric quantum mechanics. Chin. Phys. B 22, 120302 (2013)

39. Onate, C.A., Ojonubah, J.O.: Relativistic and Non-relativistic solutions of the generalized Poschl-Teller and Hyperbolical potentials with some Thermodynamic properties. Int. J. Mod. Phys. E 24, 1550020 (2015)

40. Onate, C.A., Oyewumi, K.J., Falaye, B.J.: Approximate Solutions of the Schrodinger with the Hyperbolical potential: Supersymmetric Approach. Few-Body Syst. 55, 61 (2014)

41. Hassanabadi, H., Maghsoodi, E., Zarrinkamar, S.: Dirac equation with vector and scalar cornell potentials and an external magnetic field. Ann. der Physik. Ann. der Phys. 525, 944 (2013)

42. Hassanabadi, H., Maghsoodi, E., Zarrinkamar, S.: Relativistic symmetries of Schioberg and general Manning-Rosen potentials. Commun. Theor. Phys. 58, 807 (2012)

43. Hamzavi, M., Thylwe, K.E., Rajabi, A.A.: Approximate Bound states solution of the Hellmann potential. Commun. Theor. Phys. 60, 1 (2013) 